\title{
TRANSFERENCIA DEL GEN $L r 14 a$ DE TRIGOS HARINEROS A TRIGOS CRISTALINOS Y EXPRESIÓN DE LA RESISTENCIA A ROYA DE LA HOJA
}

\author{
TRANSFERING THE Lr14a GENE FROM BREAD WHEAT TO DURUM AND ITS EXPRESSION \\ AGAINST LEAF RUST
}

\author{
Julio Huerta Espino ${ }^{1 *}$, Ravi P. Singh ${ }^{2}$, H. Eduardo Villaseñor Mir ${ }^{1}$, Ernesto Solís Moya ${ }^{3}$, Eduardo Espitia \\ Rangel $^{1}$ y S. Gerardo Leyva Mir $^{4}$
}

\begin{abstract}
${ }^{1}$ Programa de Trigo y Avena, Campo Experimental Valle de México, Instituto Nacional de Investigaciones Forestales Agrícolas y Pecuarias (INIFAP). Apdo. Postal 10. 56230, Chapingo, Estado de México. Tel: 01(595) 9543536 Ext. 127 y Fax (595) 9546528. ${ }^{2}$ Centro Internacional de Mejoramiento de Maíz y Trigo (CIMMYT). Apdo. Postal 6-641. 06600 México DF, México. ${ }^{3}$ INIFAP. Campo Experimental Bajío. Apdo. 112. 38000, Celaya Guanajuato, México. ${ }^{4}$ Depto. de Parasitologia, Universidad Autónoma Chapingo. 56230, Chapingo, Estado de México.

* Autor para correspondencia (j.huerta@cgiar.org)
\end{abstract}

\section{RESUMEN}

El origen del gen de resistencia $L r 14 a$ a roya de la hoja causada por Puccinia triticina E. es el trigo tetraploide 'Yaroslav emmer' (Triticum dicoccum L.). En México, todas las razas de trigo harinero (T. aestivum) importantes son virulentas a este gen. Sin embargo, las razas que atacan trigos cristalinos o duros son avirulentas a $\mathrm{Lr} 14 \mathrm{a}$. Se ha determinado la presencia de $L r 14 a$ en trigo harinero y en especies silvestres tetraploides, pero no en trigos cristalinos o trigos duros ( $T$. turgidum spp durum). El gen Lr14a se transfirió a la variedad cristalina 'Altar C84' a partir de la cruza de ésta con la línea monogénica de trigo harinero 'RL6013' (Selkirk/6*Thatcher) y una retrocruza hacia 'Altar C84'. Para determinar la genética de la resistencia de la variedad 'Jupare C2001', resistente a la raza de roya de la hoja que venció la resistencia de 'Altar $\mathrm{C84}^{\prime}$ ', y determinar si 'Jupare C2001' posee Lr14a, se cruzó con 'Altar C84' y con 'Altar C84' + Lr14a. La respuesta del gen de resistencia a roya de la hoja $L r 14 a$ a la infección en plántula a las razas BBG/BN y BCG/BN que son virulentas en 'Altar C84', es de heterogénea con uredinios grandes $\left(X\right.$ a $\left.X^{+}\right)$en la escala de 0-4, tanto en trigos harineros como en 'Altar C84', mientras que confiere casi inmunidad en planta adulta a estas mismas razas. La cruza de 'Jupare C2001' con 'Altar C84' + Lr14a indicó que 'Jupare C2001' no posee $L r 14 a$, y que la resistencia de esta última se basa en dos genes complementarios dominantes. Para el funcionamiento efectivo de la resistencia que el gen $\mathbf{L r 1 4 a}$ confiere en trigos cristalinos en respuesta a las razas de roya de la hoja que preferentemente atacan trigos harineros, es necesaria la presencia del gen de 'Altar C84'.

Palabras clave: Triticum spp. Puccinia triticina, genes de resistencia, genes complementarios, cruzas inter-específicas.

\section{SUMMARY}

The origin of the resistance gene $L r 14 a$ against leaf rust (Puccinia triticina E.) is the tetraploid wheat 'Yaroslav emmer' (Triticum dicoccum L.). In Mexico, all the important rust races attacking bread wheat (T. aestivum L.) are virulent to this gene. However, races attacking durum wheat are avirulent to $L r 14 a$. The presence of $L r 14 a$ in several bread wheat cultivars and wild tetraploid species have been determined, but not in durum wheat. The $L r 14 a$ gene was transferred to the cultivar 'Altar C84' using the monogenic bread wheat line 'RL6013' (Selkirk/6*Thatcher) with the durum wheat 'Altar C84' and a backcross to 'Altar C84'. In order to determine the genetics of the resistance of the durum wheat cultivar 'Jupare C2001' resistant to the leaf rust race that overcome the 'Altar C84' resistance, and to determine if 'Jupare C2001' carries Lr14a, 'Jupare C2001' was crossed with 'Altar C84' and 'Altar C84' $+L r 14 a$. The response of $L r 14 a$ to the infection of leaf rust in seedlings to races $B B G / B N$ and $B C G / B N$ which are virulent to 'Altar C84' is heterogeneous whit large urenidiums $\left(\mathrm{X}\right.$ to $\mathrm{X}^{+}$in the 0-4 scale) in both bread and durum 'Altar C84' background. In adult plant stage to the same races, Lr14a confers near immunity. For better and effective expression of the resistance that $\mathrm{Lr} 14 \mathrm{a}$ gene confers against the leaf rust races preferably attacking bread wheat, is necessary the 'Altar C84' gene.

Index words: Triticum spp., Puccinia triticina, resistance genes, complementary genes, inter-specific crosses.

\section{INTRODUCCIÓN}

$\mathrm{Al}$ inicio del mejoramiento genético del trigo harinero y cristalino para resistencia a roya de la hoja (Puccinia triticina E.) se creía que el nivel de resistencia se incrementaría conforme se redujera el nivel de haploidía, porque se consideraba que los trigos diploides eran más resistentes que los tetraploides, y éstos a su vez más resistentes que los hexaploides. En la actualidad se sabe que cada especie de trigo tiene su propia población de roya de la hoja, y que existe especialización fisiológica de esta roya en trigos tetraploides (Huerta-Espino y Roelfs, 1988, 1989) y en otras especies (Anikster et al., 1997).

Se han catalogado 54 genes que confieren resistencia a la roya de la hoja en trigo harinero (McIntosh et al., 1995), de los cuales, solamente $L r 14 a, 23$ y 33 se han 
identificado en trigos tetraploides; recientemente, el gen Lr53 fue transferido de $T$. dicoccoides a trigos harineros (Marais et al., 2005). Además, se ha postulado la presencia en los trigos harineros de los genes Lr16 y Lr17a (Zhang y Knott, 1990), por el tipo de infección que causan pero no se han confirmado genéticamente. Otros genes identificados en trigos cristalinos son $\mathrm{Lr} 10$ (Aguilar-Rincón et al., 2001) y Lr13 (Singh et al., 1992).

La presencia del gen Lrl4a en trigos harineros es el resultado de la cruza de 'Yaroslav Emmer' (T. dicoccum) $\mathrm{x}$ 'Marquis' (trigo harinero de primavera, T. aestivum) hecha por McFadden (1930) en 1915, de la cual sólo una planta F1 resultó fértil de modo que la selección en F2 y F3 fue difícil. En la F4 de más de 5000 plantas, fueron resistentes a la roya del tallo $(P$. graminis f. sp. tritici). La progenie de estas plantas fue seleccionada con otros criterios de selección además de la roya del tallo, como resistencia a otras enfermedades y contenido de proteína. En la generación F9, una selección fue nombrada 'Hope', la cual tenía muchos caracteres del trigo harinero. Las cruzas de 'Hope' y 'H-44' con otros genotipos dieron origen a muchas variedades resistentes a la roya del tallo en América del Norte y otros países (Chester, 1946).

De 'Hope' y 'Spica' como fuentes del gen Lr14a, se han derivado variedades como 'Regent', 'Renown', 'Mida' y 'Rival' (Chester, 1946). Lr14a también se ha identificado en las variedades 'Aotea', 'Gala', 'Glenwari' y 'Redman' (McIntosh et al., 1967); 'Selkirk' (Dyck y Samborski, 1970). Las variedades mexicanas que poseen Lr14a son 'Siete Cerros 66', 'Pitic 62', 'Pénjamo 62' y 'Esmeralda 86' (Singh y Rajaram, 1991). Otros genotipos de trigo harinero donde se ha encontrado el gen $\mathrm{Lr} 14 \mathrm{a}$ son: 'Sonalika', 'WL711' (McIntosh et al., 1995), 'Minter', 'Newthatch' y 'Scout 66' (Dyck y Samborsky, 1970).

Es probable que los genes $\operatorname{Lr} 14 a$ y $S r 17$, éste último confiere resistencia a la roya del tallo y Pm5 que confiere resistencia a la cenicilla polvorienta (Blumeria graminis $\mathrm{f}$. sp. tritici), hayan sido transferidos a trigos harineros a partir de 'Hope' y de 'H-44'. Lr14a aún se encuentra en la mayoría de trigos harineros que poseen al gen $\operatorname{Sr} 17$ (McIntosh et al., 1995).

En la actualidad, el uso del gen $L r 14 a$ en trigos harineros ha sido restringido porque la mayoría de aislamientos de Puccinia triticina han resultado virulentos a pesar de la presencia de este gen, con algunas excepciones en Estados Unidos (Kolmer et al., 2006), Canadá (McCallum y SetoGoh, 2002), en varios países de la Comunidad Europea en países nórdicos (Hysing et al., 2006).
Aparte de 'Yaroslav Emmer', no existe evidencia de la presencia de Lr14a en trigos duros o cristalinos u otras especies tetraploides. Desafortunadamente la variedad 'Yaroslav Emmer', donde se supone se identificó este gen (McFadden, 1930), ya no existe, y la variedad 'Marquis' no lo posee (McIntosh et al., 1995).

Algunas poblaciones de roya de la hoja han mostrado cierta preferencia por trigos duros o cristalinos, y son diferentes a la roya de la hoja que ataca a trigos harineros, (Huerta-Espino y Roelfs, 1992; Singh, 1991, Singh et al., 2004, Ordóñez y Kolmer, 2007), aun cuando esta última pertenece a la misma forma especial $P$. triticina E., de acuerdo con Anikster et al. (1997). En cuanto a su fórmula de virulencia/avirulencia las razas del hongo difieren entre sí; en particular las que atacan trigos cristalinos aparentemente son mas avirulentas que las que atacan a trigos harineros, basados en su reacción de plantas diferenciales (Singh, 1991).

En el noroeste de México apareció en 2001 una nueva raza de roya de la hoja, $\mathrm{BBG} / \mathrm{BN}$ (según la nomenclatura de Singh, 1991), la cual venció la resistencia de las variedades de trigo cristalino que incluyen a 'Altar C85' que ocupaba más de $85 \%$ de la superficie sembrada de trigo, así como a las variedades 'Atil C2000', 'Nacori C97' y 'Aconchi C89'. Esta nueva raza causó pérdidas económicas de más de 35 millones de dólares tanto por la aplicación de fungicidas como por las pérdidas potenciales de $10 \%$ al no poder sembrar la variedad 'Atil C2000'; además, hubo pérdidas en el mercado internacional por no poder exportar el trigo con la calidad industrial requerida (Singh et al., 2004).

Como consecuencia de la pérdida de la resistencia en las variedades comerciales, se determinó liberar a la línea Stot//Altar C84/Alondra como 'Jupare C2001'; la cual llegó a ocupar $95 \%$ de la superficie cultivada con trigos cristalinos hasta el ciclo 2007-2008, cuando se volvió susceptible por la aparición de una nueva raza de roya de la hoja (Huerta-Espino et al., 2009). Por la importancia de la resistencia a la roya de la hoja en trigos cristalinos del noroeste de México, se decidió transferir el gen Lr14a de trigos harineros a trigos cristalinos así como determinar la genética de la resistencia en 'Jupare C2001' y la expresión fenotípica del gen $\operatorname{Lr} 14 a$ en trigos cristalinos, y verificar si 'Jupare C2001' posee el gen Lr14a.

\section{MATERIALES Y MÉTODOS}

\section{Material genético}

Nueve genotipos de trigo harinero (Cuadro 1) que poseen el gen $L r 14 a$ (McIntosh et al., 1995) y la variedad 
de trigo cristalino 'Jupare C2001', en la cual se postuló la presencia de $L$ rl $4 a$, fueron sembrados en macetas de plástico y las plantas fueron inoculadas con la raza $\mathrm{BBG} / \mathrm{BN}$ de roya de la hoja que ataca preferentemente a trigos cristalinos tanto en plántula como en planta adulta.

Cuadro 1. Respuesta a la inoculación de genotipos de trigo harinero en que se ha postulado la presencia del gen de resistencia $L r 14 a$ en contra de la raza $B B G / B N$.

\begin{tabular}{lcc}
\hline & \multicolumn{2}{c}{ Tipo de infección } \\
\cline { 2 - 3 } Variedad & Plántula & Planta adulta \\
'Siete Cerros T66' & $;$ & 0 \\
'Comb VII (Sr13+Sr17)' & $;, \mathrm{X}$ & 0 \\
'Sr H' & $\mathrm{X}$ & 0 \\
'RL 6013' & $\mathrm{X}$ & 0 \\
'Spica' & $\mathrm{X}$ & 0 \\
'LC/Kenya Hunter (Sr17)' & $;$ & 0 \\
'Renown' & $;$ & 0 \\
'Hope' & $; 1$ & 0 \\
'Selkirk' & $\mathrm{X}$ & 0 \\
'Jupare C2001' & $\mathrm{X}$ & 0 \\
\hline Escala del 0-4, de acuerdo con Roelfs et al. (1992): $\mathbf{0}=$ inmune; \\
= casi inmune; $\mathbf{1}=$ muy resistente; y X = heterogénea.
\end{tabular}

\section{Transferencia del gen $\mathrm{LrI4} a$ de trigo harinero a trigo cristalino}

El trigo harinero 'Thatcher $L r 14 a$ ' (Selkirk/6*Thatcher = RL6013), se usó como progenitor femenino y se cruzó con el trigo cristalino 'Altar C84'; de la cruza se obtuvieron 40 semillas $\mathrm{F} 1$ que fueron sembradas en invernadero en 10 macetas con cuatro plantas por maceta. Todas las plantas F1 se retrocruzaron con 'Altar C84' y se obtuvieron 100 semillas de la retrocruza F1RC1, como se ilustra en la Figura 1.

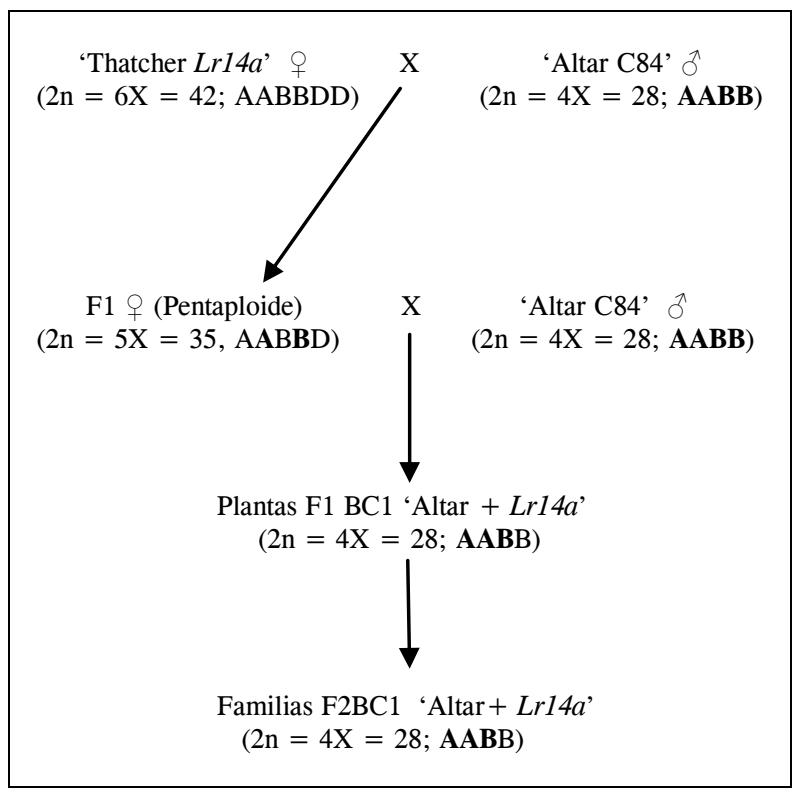

Figura 1. Esquema de cruzamientos para transferir el gen Lr14a de resistencia a roya de la hoja de trigos harineros a trigos cristalinos.
En esta generación se esperaba una proporción 1:1 de plantas resistentes y susceptibles. Las 100 plantas resultantes de la F1RC1 fueron inoculadas con la raza $\mathrm{BBG} / \mathrm{BN}$, y las plantas resistentes y fértiles con características de trigo cristalino se seleccionaron para avanzar a la siguiente generación. En F2RC1 se seleccionaron 11 plantas con buenas características agronómicas y de tipo cristalino, que luego en las F3RC1 originaron 36 familias que se inocularon en invernadero con la raza $\mathrm{BBG} / \mathrm{BN}$ de roya de la hoja, tanto en plántula como en planta adulta, además de los testigos 'Jupare C2001', 'Altar C84', 'Atil C2000' y 'Thatcher Lr14a'. Estos estudios se hicieron en invernadero en el Centro Internacional de Mejoramiento de Maíz y Trigo (CIMMYT), estación experimental El Batán, Estado de México ( $19^{\circ} 31^{\prime}$ LN y $99^{\circ} 53^{\prime}$ LO).

\section{Cruzas resistente $\mathbf{x}$ susceptible}

Altar $+\boldsymbol{L r 1 4 a / A l t a r}$ C84. Una vez que se obtuvieron familias homocigóticas resistentes, dos de ellas (Altar 2*/TcLr14a 9.3.3 y Altar 2*/TcLr14a 9.3.4) se cruzaron con la variedad susceptible 'Altar C84' con el fin de determinar si la resistencia presente en 'Altar $+L r 14 a$ ' era debida a la acción de uno o más genes.

Altar C84/Jupare C2001. Esta cruza se hizo para determinar la genética de la resistencia de 'Jupare C2001'. En esta cruza 'Altar C84' se usó como progenitor femenino y 'Jupare C2001' como progenitor masculino.

\section{Cruzas resistente $\mathrm{x}$ resistente}

Altar + Lr14a/Jupare C2001. Las líneas Altar $2 * / \mathrm{Tc} L r 14 a 9.3 .3$ y Altar $2 * / \mathrm{Tc} L r 14 a 9.3 .4$ se cruzaron con la variedad 'Jupare C2001', debido al tipo de infección mostrado por esta variedad, que es del tipo mesotético (X) en la escala del 0 al 4 donde: 0 (inmune), ; (casi inmune), 1 (muy resistente), $\mathbf{2}$ (moderadamente resistente), $\mathbf{X}$ (heterogénea o mesotética), 3 (moderadamente susceptible) y 4 (susceptible) según Roelfs et al., (1992), muy similar al tipo de infección mostrado por el gen Lr14a. Esta cruza se hizo para determinar si la resistencia en 'Jupare C2001' es condicionada por Lr14a.

En todas las cruzas la F1 se sembró a una distancia entre planta de $30 \mathrm{~cm}$, y la progenie de tres plantas se avanzó hasta obtener familias F3. La F1 se sembró en los invernaderos del CIMMYT ubicados en El Batán, Estado de México, y la progenie de las tres plantas F1 se sembraron en Celaya Gto. en el Campo Experimental Bajío (CEBAJ) del Instituto Nacional de Investigaciones Forestales, Agrícolas y Pecuarias (INIFAP) $\left(20^{\circ} 32^{\prime} \mathrm{LN}\right.$ 
y $100^{\circ} 48^{\prime}$ LO) durante el ciclo Otoño-Invierno 20032004 para obtener la semilla F3.

\section{Evaluación de la enfermedad y pruebas de resistencia}

Plántula en invernadero. En otoño del 2004, se evaluó en invernadero la F3 en estado de plántula con la raza $\mathrm{BBG} / \mathrm{BN}$ de roya de la hoja con virulencia para la variedad 'Altar C84', que posee la siguiente fórmula de avirulencia/virulencia: $L r 1,2 a, 2 b, 2 c, 3,3 k a, 3 b g, 9$, $13,14 a, 15,16,17,18,19,21,24,26,27+31,29,32$, $35,36 / 10,11,20,23, y 33$, de acuerdo con Singh et al. (2004).

Planta adulta en campo. Las familias F3 de cada cruza fueron sembradas en campo durante el verano del 2005 en el Batán, Texcoco, Estado de México, en un surco doble de $1 \mathrm{~m}$ de longitud por familia. Las parcelas se rodearon con bordos de la variedad susceptible 'Atil C2000'. Las plantas de los bordos susceptibles se inocularon con la raza BBG/BN a los $28 \mathrm{~d}$ de edad. Con base en los niveles de infección, las familias F3 se clasificaron en: Homocigóticas resistentes, Segregantes y Homocigóticas susceptibles, tanto en las pruebas de plántula en invernadero como en planta adulta en el campo, cuando la severidad de la roya de la hoja en el progenitor suscep-tible 'Altar C84' alcanzó entre 70 y 80 $\%$ de infección, de acuerdo con Roelfs et al. (1992). Las proporciones de familias se sometieron a una prueba de $X^{2}$ para comparar las frecuencias observadas con las frecuencias teóricas esperadas, y así determinar el número de genes involucrados en cada cruza.

\section{RESULTADOS Y DISCUSIÓN}

Transferencia del gen $\mathrm{Lr} 14 \mathrm{a}$ de trigo harinero a trigo cristalino. En la generación F2RC1 se identificaron tres plantas resistentes (8R, 9R y 11R) con buen tipo agronómico y con todas las características de trigos cristalinos. Los niveles de infección en planta adulta en $8 \mathrm{R}$ y $9 \mathrm{R}$ fueron de casi inmune (;) a inmune (0); y para la $11 \mathrm{R}$ de casi inmune (;) a muy resistente (1). Todas las progenies $\mathrm{F} 3 \mathrm{RC} 1$ de $8 \mathrm{R}$ y $9 \mathrm{R}$ fueron resistentes en plántula, mientras que en $11 \mathrm{R}$ segregaron nueve de sus 23 progenies, 13 fueron resistentes y una susceptible (Cuadro $2)$. Por el contrario, en planta adulta únicamente las progenies de $9 \mathrm{R}$ fueron todas resistentes.

La introgresión del gen $\operatorname{Lr} 14 a$ fue posible debido a que la variedad 'Thatcher' (ne1.ne1,ne2.ne2) que es el fondo genético del gen Lr14a, carece de los dos genes dominantes Ne1.Ne1, o Ne2.Ne2 (Hermsen, 1963). Cuando estos genes se encuentran en forma dominante en un genotipo, causa lo que se conoce como necrosis híbrida que ocasiona la muerte de la F1. Es posible que 'Thatcher' combine bien con trigos cristalinos debido a que esta variedad es producto de una cruza del trigo cristalino 'Iumillo' con los trigos harineros 'Marquis' y 'Kanred' (Luig, 1983).

En ninguna cruza de Thatcher Lr14a/Altar C84 fue necesario hacer rescate de embriones y cultivos para inducir la formación de raíces y tallos, pero la cruza recíproca no produjo semilla. En este tipo de cruzamientos donde existe un desbalance en el número cromosómico, es frecuente que la F1 sea androestéril, como ocurrió en este caso. Por tanto, para poder obtener progenie fértil es necesario que la $\mathrm{F} 1$ sea retrocruzada con el progenitor de trigo cristalino, que en este caso es la variedad 'Altar C84'.

Cruzas resistente $\mathbf{x}$ susceptible. La cruza Altar + Lr14a/Altar C84 mostró una herencia monogénica de 1:2:1 de familias F3 resistentes, segregantes y susceptibles, respectivamente, tanto en plántula como en planta adulta (Cuadro 3). El gen de resistencia implicado en esta cruza, $L r 14 a$, fue confirmado por Herrera-Foessel et al. (2008) mediante análisis con marcadores moleculares SST Xwgm344-7B y Xgwm146-7B que claramente discriminan a la variedad 'Thatcher' de Thatcher +Lr14a (RL6013), así como distinguen a la variedad 'Altar C84' de Altar+Lr14a. El tipo de infección de $L r 14 a$ en trigos cristalinos no difiere del que se expresa en trigos harineros (RL6013) en estado de plántula. También en planta adulta la respuesta de las plantas que contienen el gen $L r 14 a$ es de inmunidad (sin signos visibles de infección), tanto en trigos cristalinos como en trigos harineros.

En México es fácil distinguir dos poblaciones de roya de la hoja; una tiene como hospedantes primarios a trigos harineros, pero son avirulentos a la mayoría de los trigos cristalinos, y la otra preferentemente ataca a trigos cristalinos pero es avirulenta a la mayoría de las variedades de trigo harinero (Huerta-Espino y Roelfs, 1992; Singh, 1991, Singh et al., 2004). Con excepción de la raza $\mathrm{BBB} / \mathrm{BB}$, todas las razas de trigo harinero identificadas son virulentas a $\operatorname{Lr} 14 a$, por lo que el uso de este gen en trigos harineros es limitado. Sin embargo, Lr14a es muy efectivo en contra de poblaciones de roya de la hoja en trigos cristalinos. Por tanto, Lr14a debe estar en un fondo genético que contenga además el gen de 'Altar C84' (aún no catalogado) entre otros, para que sea efectivo en contra de las razas de roya de la hoja provenientes de trigos harineros. 


\begin{tabular}{|c|c|c|c|c|}
\hline Núm. de Fam. & Cruza o variedad & Origen & Plántula $^{\dagger}$ & Planta adulta $^{\dagger \dagger}$ \\
\hline 1 & Altar $2 * / \operatorname{TcLr14a}$ & 8.1 .1 & $\mathrm{R}$ & $\mathrm{R}$ \\
\hline 2 & Altar $2 * / \operatorname{Tc} L r 14 a$ & 8.1 .2 & $\mathrm{R}$ & $\mathrm{R}$ \\
\hline 3 & Altar $2 * / \operatorname{Tc} L r 14 a$ & 8.1 .3 & $\mathrm{R}$ & $\mathrm{R}$ \\
\hline 4 & Altar $2 * / \operatorname{Tc} L r 14 a$ & 8.1 .4 & $\mathrm{R}$ & $\mathrm{R}$ \\
\hline 5 & Altar $2 * / \operatorname{Tc} L r 14 a$ & 8.2 .1 & $\mathrm{R}$ & SEG \\
\hline 6 & Altar $2 * / \operatorname{TcLr} 14 a$ & 8.2 .2 & $\mathrm{R}$ & SEG \\
\hline 7 & Altar $2 * / \operatorname{Tc} L r 14 a$ & 8.2 .3 & $\mathrm{R}$ & $\mathrm{R}$ \\
\hline 8 & Altar $2 * / \operatorname{Tc} L r 14 a$ & 8.2 .4 & $\mathrm{R}$ & $\mathrm{R}$ \\
\hline 9 & Altar $2 * / \operatorname{TcLr} 14 a$ & 8.2 .5 & $\mathrm{R}$ & $\mathrm{R}$ \\
\hline 10 & Altar $2 * / \operatorname{Tc} L r 14 a$ & 9.3 .1 & $\mathrm{R}$ & $\mathrm{R}^{\dagger \dagger \dagger}$ \\
\hline 11 & Altar $2 * / \operatorname{Tc} L r 14 a$ & 9.3 .2 & $\mathrm{R}$ & $\mathrm{R}^{\dagger \dagger \dagger}$ \\
\hline 12 & Altar $2 * / \operatorname{Tc} L r 14 a$ & 9.3 .3 & $\mathrm{R}$ & $\mathrm{R}^{\uparrow}$ \\
\hline 13 & Altar $2 * / \operatorname{Tc} L r 14 a$ & 9.3 .4 & $\mathrm{R}$ & $\mathrm{R}^{\prime}$ \\
\hline 14 & Altar $2 * / \operatorname{Tc} L r 14 a$ & 11.4 .1 & SEG & SEG \\
\hline 15 & Altar $2 * / \operatorname{Tc} L r 14 a$ & 11.4 .2 & SEG & SEG \\
\hline 16 & Altar $2 * / \operatorname{Tc} L r 14 a$ & 11.4 .3 & $\mathrm{R}$ & $\mathrm{R}$ \\
\hline 17 & Altar $2 * / \operatorname{Tc} L r 14 a$ & 11.4 .4 & SEG & $\mathrm{S}$ \\
\hline 18 & Altar $2 * / \operatorname{Tc} L r 14 a$ & 11.5 .1 & SEG & $\mathrm{S}$ \\
\hline 19 & Altar $2 * / \operatorname{Tc} L r 14 a$ & 11.5 .2 & $\mathrm{R}$ & $\mathrm{R}$ \\
\hline 20 & Altar $2 * / \operatorname{Tc} L r 14 a$ & 11.5 .3 & $\mathrm{R}$ & $\mathrm{R}$ \\
\hline 21 & Altar $2 * / \operatorname{Tc} L r 14 a$ & 11.5 .4 & $\mathrm{R}$ & SEG \\
\hline 22 & Altar $2 * / \operatorname{Tc} L r 14 a$ & 11.5 .5 & $\mathrm{R}$ & SEG \\
\hline 23 & Altar $2 * / \operatorname{Tc} L r 14 a$ & 11.6 .1 & SEG & $\mathrm{R}$ \\
\hline 24 & Altar $2 * / \operatorname{Tc} L r 14 a$ & 11.6 .2 & $\mathrm{R}$ & $\mathrm{R}$ \\
\hline 25 & Altar $2 * / \operatorname{Tc} L r 14 a$ & 11.6 .3 & SEG & SEG \\
\hline 26 & Altar $2 * / \operatorname{Tc} L r 14 a$ & 11.6 .4 & $\mathrm{R}$ & SEG \\
\hline 27 & Altar $2 * / \operatorname{TcL} L 14 a$ & 11.6 .5 & $\mathrm{R}$ & $\mathrm{R}$ \\
\hline 28 & Altar $2 * / \operatorname{Tc} L r 14 a$ & 11.7 .1 & SEG & SEG \\
\hline 29 & Altar $2 * / \operatorname{Tc} L r 14 a$ & 11.7 .2 & SEG & $\mathrm{S}$ \\
\hline 30 & Altar $2 * / \operatorname{Tc} L r 14 a$ & 11.7 .3 & $\mathrm{R}$ & $\mathrm{R}$ \\
\hline 31 & Altar $2 * / \operatorname{TcLr} 14 a$ & 11.7 .4 & $S$ & S \\
\hline 32 & Altar $2 * / \operatorname{Tc} L r 14 a$ & 11.7 .5 & $\mathrm{R}$ & $\mathrm{R}$ \\
\hline 33 & Altar $2 * / \operatorname{Tc} L r 14 a$ & 11.8 .1 & $\mathrm{R}$ & $\mathrm{R}$ \\
\hline 34 & Altar $2 * / \operatorname{Tc} L r 14 a$ & 11.8 .2 & $\mathrm{R}$ & $\mathrm{R}$ \\
\hline 35 & Altar $2 * / \operatorname{Tc} L r 14 a$ & 11.8 .3 & SEG & $\mathrm{R}$ \\
\hline 36 & Altar $2 * / \operatorname{Tc} L r 14 a$ & 11.8 .4 & $\mathrm{R}$ & SEG \\
\hline 37 & Jupare C2001 & & $\mathrm{R}$ & $\mathrm{R}$ \\
\hline 38 & Altar C84 & & $S$ & $S$ \\
\hline 39 & Atil C2001 & & S & $S$ \\
\hline 40 & Thatcher $\operatorname{Lr} 14 a$ & & $\mathrm{R}$ & $\mathrm{R}$ \\
\hline
\end{tabular}

Al estudiar las familias F3 de la cruza de Altar C84/Jupare C2001 fue difícil clasificar las familias en resistentes, segregantes y susceptibles, debido al tipo de infección (mesotético o heterogénea " $\mathrm{X}$ " = todos los tipos de infección ocurren en la misma hoja) que el progenitor resistente muestra, es casi similar al del progenitor susceptible, particularmente cuando la temperatura está por debajo de $20{ }^{\circ} \mathrm{C}$, por ello aquí se optó por usar los datos de campo en planta adulta. Los resultados indicaron una herencia para dos genes dominantes complementarios 1:8:7 de familias F3 (homocigóticas resistentes, segregantes y homocigóticas susceptibles, respectivamente). En otro estudio (HerreraFoessel et al., 2005) en que 'Jupare C2001' fue cruzado con la variedad 'Atil C2001', se encontró que la resistencia a la roya de la hoja raza $\mathrm{BBG} / \mathrm{BN}$ en 'Jupare C2001' también está condicionada por dos genes complementario dominantes, mismo resultado que en la cruza 'Jupare C2001' x 'Altar C84'. Lo anterior permite deducir que $\operatorname{Lr} 14 a$ no es el responsable de la resistencia a la roya de la hoja en 'Jupare C2001', aun cuando los tipos de infección en plántula y planta adulta sean muy similares. La base de la resistencia genética a la roya de la hoja tanto en 'Atil C2001' como en 'Altar C84', parece ser la misma porque no se observó segregación en la F2 de la cruza de éstas (Huerta-Espino, datos sin publicar). 
Cruzas resistente $\mathbf{x}$ resistente. Si la resistencia en 'Jupare C2001' no es condicionada por Lr14a, de acuerdo con lo descrito en el párrafo anterior, la proporción de familias F3 de la cruza Altar+Lr14a/Jupare C2001 debería mostrar una segregación 19:38:7 de familias homocigóticas resistentes: familias segregando: familias homocigóticas susceptibles, respectivamente. Ello correspondería a una segregación para tres genes de resistencia; dos genes complementarios dominantes provenientes de 'Jupare C2001' y uno dominante $(\operatorname{Lr} 14 a)$ proveniente de Altar + Lr14a.

La proporción esperada 19:38:7 de familias resistentes, familias segregando y familias susceptibles, respectiva- mente con respecto a las proporciones observadas, no fueron significativas (Cuadro 3). Este resultado indica la presencia de tres genes de resistencia en la cruza Altar+Lr14a/Jupare C2001, los dos genes complementarios provenientes de 'Jupare C2001' y el gen Lr14a transferido a la variedad 'Altar C84'. El par de genes complementarios conocidos en trigo son $L r 27+31$ (Singh y McIntosh, 1984), y se ha indicado que Lr31 está fuertemente ligado a $L r 12$, o que $L r 31$ es el mismo gen que Lr12 (Singh et al., 1999), conocido como un gen que confiere resistencia específica en planta adulta y que es efectivo en algunas ciertas razas de trigo harinero en México (Huerta-Espino y Singh 1996).

Cuadro 3. Cruza, número de familias F3 resistentes, segregantes y susceptibles, observadas y esperadas; relación fenotípica y prueba de $X^{2}$ de las diferentes cruzas de trigos cristalinos en respuesta a la roya de la hoja, en plántula y en planta adulta. Número de familias

\begin{tabular}{lccccccccrrr} 
& \multicolumn{1}{c}{ Total } & \multicolumn{2}{c}{ Resistentes } & \multicolumn{2}{c}{ Segregantes } & \multicolumn{2}{c}{ Susceptibles } & Relación ${ }^{\dagger \dagger}$ & $X^{2}$ & Prob. \\
\hline Cruza & & Obs. & Esp. & Obs. & Esp. & Obs. & Esp. & & \\
Altar+Lr14a/Altar C84 & 184 & 48 & 46 & 94 & 92 & 42 & 46 & $1: 2: 1$ & 0.478 & $0.80-0.70$ \\
Altar+Lr14a/Altar C84 & 184 & 50 & 46 & 100 & 92 & 34 & 46 & $1: 2: 1$ & 4.174 & $0.20-0.05$ \\
& & & & & & & & & \\
Altar+Lr14a/Jupare C2001 & 198 & 50 & 58.78 & 126 & 117.56 & 22 & 21.65 & $19: 38: 7$ & 1.923 & $0.70-0.50$ \\
Altar C84/Jupare C2001 & 148 & 11 & 9.25 & 72 & 74 & 65 & 64.75 & $1: 8: 7$ & 0.386 & $0.95-0.80$
\end{tabular}

${ }^{\dagger}$ Numerador, Plántula-invernadero; Denominador, Planta adulta-Campo.

${ }^{\dagger}$ Relación 1:2:1 = segregación esperada para un gen de resistencia con dominancia completa.

Relación 1:8:7 = segregación esperada para dos genes complementarios dominantes.

Relación 19:38:7 = segregación esperada para tres genes; dos dominantes complementarios y un tercer gen con dominancia completa.

Cuadro 4. Genotipos F3 con características agronómicas deseables de trigos cristalinos portadoras del gen $\operatorname{Lr} 14 a$ y resistentes en plántula en invernadero y en planta adulta en campo.

\begin{tabular}{|c|c|c|c|c|c|}
\hline $\begin{array}{l}\text { Num. de } \\
\text { Familia } \\
\end{array}$ & Cruza & Plántula $^{\dagger}$ & $\mathrm{Campo}^{\dagger}$ & Color de grano & Tipo \\
\hline 105 & Altar + Lr14a/Altar C84 & $\bar{R}$ & $\bar{R}$ & Rojo & $* *$ \\
\hline 115 & Altar $+L r 14 a /$ Altar C84 & $\mathrm{R}$ & $\mathrm{R}$ & Rojo & $* *$ \\
\hline 116 & Altar + Lr14a/Altar C84 & $\mathrm{R}$ & $\mathrm{R}$ & Rojo & $* *$ \\
\hline 139 & Altar + Lr14a/Altar C84 & $\mathrm{R}$ & $\mathrm{R}$ & Amarillo & $* *$ \\
\hline 153 & Altar $+L r 14 a /$ Altar C84 & $\mathrm{R}$ & $\mathrm{R}$ & Amarillo & $* *$ \\
\hline 170 & Altar + Lr14a/Altar C84 & $\mathrm{R}$ & $\mathrm{R}$ & Rojo & $* *$ \\
\hline 171 & Altar $+L r 14 a /$ Altar C84 & $\mathrm{R}$ & $\mathrm{R}$ & Rojo & $* *$ \\
\hline 192 & Altar + Lr14a/Altar C84 & $\mathrm{R}$ & $\mathrm{R}$ & Seg. color & $* *$ \\
\hline 201 & Altar $+L r 14 a /$ Jupare C2001 & $\mathrm{R}$ & $\mathrm{R}$ & Amarillo & \\
\hline 209 & Altar + Lr14a/Jupare C2001 & SEG & $\mathrm{R}$ & Amarillo & \\
\hline 214 & Altar + Lr14a/Jupare C2001 & $\mathrm{R}$ & $\mathrm{R}$ & Amarillo & \\
\hline 215 & Altar + Lr14a/Jupare C2001 & $\mathrm{R}$ & $\mathrm{R}$ & Amarillo & \\
\hline 216 & Altar +Lr14a/Jupare C2001 & $\mathrm{R}$ & $\mathrm{R}$ & Amarillo & \\
\hline 218 & Altar +Lr14a/Jupare C2001 & $\mathrm{R}$ & $\mathrm{R}$ & Amarillo & \\
\hline 228 & Altar +Lr14a/Jupare C2001 & $\mathrm{R}$ & $\mathrm{R}$ & Amarillo & \\
\hline 238 & Altar +Lr14a/Jupare C2001 & $\mathrm{R}$ & $\mathrm{R}$ & Rojo & \\
\hline 244 & Altar +Lr14a/Jupare C2001 & $\mathrm{R}$ & $\mathrm{R}$ & Amarillo & \\
\hline 249 & Altar +Lr14a/Jupare C2001 & $\mathrm{R}$ & $\mathrm{R}$ & Amarillo & \\
\hline 259 & Altar +Lr14a/Jupare C2001 & $\mathrm{R}$ & $\mathrm{R}$ & Rojo & \\
\hline 269 & Altar+Lr14a/Jupare C2001 & $\mathrm{R}$ & $\mathrm{R}$ & Rojo & \\
\hline 270 & Altar +Lr14a/Jupare C2001 & $\mathrm{R}$ & $\mathrm{R}$ & Seg. color & \\
\hline 273 & Altar+Lr14a/Jupare C2001 & $\mathrm{R}$ & $\mathrm{R}$ & Rojo & \\
\hline 276 & Altar +Lr14a/Jupare C2001 & $\mathrm{R}$ & $\mathrm{R}$ & Rojo & \\
\hline 285 & Altar +Lr14a/Jupare C2001 & $\mathrm{R}$ & $\mathrm{R}$ & Amarillo & \\
\hline
\end{tabular}


El progenitor donador de LrI4a es un trigo harinero rojo, con una característica importante en la calidad del grano de trigos cristalinos que es la presencia del pigmento amarillo. Algunas familias identificadas como resistentes son de color de grano amarillo, otras segregaron los colores rojo y amarillo, y otras sólo fueron de color rojo, este último indeseable en trigos cristalinos. Dentro de las familias F3 resistentes de la cruza Altar + Lr14a/Altar C84, se identificaron ocho familias con buen tipo agronómico y dos en particular con buen pigmento amarillo $\mathrm{y}$ alto potencial de rendimiento (Cuadro 4). En las cruzas de Altar +Lr14a/Jupare C2001 se identificaron 10 familias resistentes en planta adulta y con grano amarillo (Cuadro 4). Algunas de estas líneas poseen los genes complementarios dominantes de 'Jupare C2001' además del gen Lr14a, combinación que puede ser más efectiva que cuando se encuentran separados como en 'Altar Lr14a' o en 'Jupare C2001'.

El comportamiento del gen Lr14a en 'Altar C84' en contra de la raza BBG/BN identificada en el 2001 (Singh et al., 2004), es similar al comportamiento en los trigos harineros, donde " $\mathrm{X}$ " es el tipo de infección en la escala del 0 al 4 (Roelfs et al., 1992) y también en el fondo genético de trigos cristalinos.

En planta adulta LrI4a confiere inmunidad tanto en trigos cristalinos como en trigos harineros en presencia de la raza BBG/BN. El gen de resistencia Lr14a puede ser usado en combinación con otros genes de efectos mayores y con genes de resistencia no específica, en un programa de mejoramiento de trigo cristalino para obtener resistencia durable a la roya de la hoja.

\section{CONCLUSIONES}

Se logró la transferencia del gen de resistencia $\operatorname{Lr} 14 a$ de trigos harineros a trigos cristalinos, cuya expresión fenotípica en estos últimos es la misma que en los trigos harineros en respuesta a la infección causada por la roya de la hoja del trigo. La variedad 'Jupare C2001' no posee el gen de resistencia $L r 14 a$, sino dos genes dominantes complementarios. El gen $\mathrm{Lr} 14 a$ todavía es efectivo en contra de las razas $\mathrm{BBG} / \mathrm{BN}$ y $\mathrm{BBG} / \mathrm{BP}$ de roya de la hoja.

\section{BIBLIOGRAFÍA}

Aguilar-Rincón V H, R P Singh, G F Castillo, J Huerta-Espino (2001) Genes de resistencia a la roya de la hoja en un trigo sintético hexaploide. Rev. Fitotec. Mex. 24:161-169.

Anikster Y, W R Bushnell, T Eilam, J Manisterski, A P Roelfs (1997) Puccinia recondita causing leaf rust on cultivated wheats, wild wheats, and rye. Can. J. Bot. 75:2082-2096.
Chester K S (1946) The nature and prevention of the cereal rusts as exemplified in the leaf rust of wheat. Chronica Botanica, Walthan, Mass. 269 p.

Dyck P L, D J Samborski (1970) The genetics of two alleles for leaf rust resistance at the Lr14 locus in wheat. Can. J. Genet. Cytol. 12:689-694.

Herrera-Foessel S A, R P Singh, J Huerta-Espino, J Yuen, A Djurle (2005) New genes for leaf rust resistance in CIMMYT durum wheats. Plant Dis. 89:809-814.

Herrera-Foessel S A, R P Singh, J Huerta-Espino, H M William, V Garcia, A Djurle, J Yuen (2008). Identification and molecular characterization of leaf rust resistance gene $\mathrm{Lr} 14 \mathrm{a}$ in durum wheat. Plant Dis. 92:469-473.

Hermsen J G (1963) Hybrid necrosis as a problem for the wheat breeder. Euphytica 12:1-16.

Huerta-Espino J, A P Roelfs (1988) Puccinia recondita avirulent on wheat cultivar Thatcher. Phytopathology 78:1596.

Huerta-Espino J, A P Roelfs (1989) Physiological specialization of leaf rust on durum wheat. Phytopathology 79:1218

Huerta-Espino J, A P Roelfs (1992) Leaf rust on durum wheats. Vortr, Pflanzenzuechtg 24:100-102.

Huerta-Espino J, R P Singh (1996) Misconceptions on the durability of some adult leaf rust resistance genes in wheat. In: Proc. 9th European and Mediterranean Cereal Rust and Powdery Mildews Conference. G H J Kema, R E Niks, R A Daamen (eds). September 2-6, 1996. Lunteren, The Netherlands. pp:109-111.

Huerta-Espino J, R P Singh, Herrera-Foessel S A, J B Perez-Lopez, P Figueroa-Lopez (2009) First Detection of virulence in Puccinia triticina to resistance genes $L r 27+31$ present in durum wheat in Mexico. Plant Dis. 93:110.

Hysing S, R P Singh, J Huerta-Espino, A Merker, E Liljeroth, O Diaz (2006) Leaf rust (Puccinia triticina) resistance in wheat (Triticum aestivum) cultivars grown in Northern Europe 1992 2002. Hereditas 143:1-14.

Kolmer J A, D L Long, M E Hughes (2006) Physiologic specialization of Puccinia triticina on wheat in the United States in 2004. Plant Dis. 90:1219-1224.

Marais G F, Z A Pretorius, C R Wellings, B McCallum, A S Marais (2005) Leaf rust and stripe rust resistance genes transferred to common wheat from Triticum dicoccoides. Euphytica 143:115123.

McCallum B D, P Seto-Goh (2002) Physiologic specialization of wheat leaf rust (Puccinia triticina) in Canada in 1999. Can. J. Plant Pathol. 24: 205-210.

McFadden E S (1930) A successful transfer of emmer characters to vulgare wheat. J. Am. Soc. Agron. 22:1020-1034.

McIntosh R A, N H Luig, E P Baker (1967) Genetic and Cytogenetic studies of stem rust, leaf rust, and powdery mildew resistance in hope and related wheat cultivars. Austr. J. Biol. Sci. 20:1181-1192.

McIntosh R A, C R Wellings, R F Park (1995) Wheat rusts: An Atlas of Resistance Genes. CSIRO. Australia. 200 p.

Luig N H (1983) A Survey of Virulence Genes in Wheat Stem Rust, Puccinia graminis f.sp.tritici. Advances in Plant Breeding. Supplement 11 to Journal of Plant Breeding. 199 p.

Ordóñez M E, J A Kolmer (2007) Virulence phenotypes of a worldwide collection of Puccinia triticina from durum wheat. Phytopathology 97:344-351.

Roelfs A P, R P Singh, E E Saari (1992) Rust Diseases of Wheat: Concepts and Methods of Disease Management. CIMMYT, Mexico, DF. 81 p.

Singh D, R F Park, R A McIntosh (1999) Genetic relationship between the adult plant resistance gene Lr12 and the complementary gene $L r 31$ for seedling resistance to leaf rust in common wheat. Plant Pathol. 48:567-573. 
Singh H, H S Dhaliwal, K S Gill (1992) Diversity for leaf rust resistance in Triticum durum germplasm. Cer. Rus. Pow. Mil. Bull. 20:62-67.

Singh R P (1991) Pathogenicity variations of Puccinia recondita f. sp. tritici and $P$. graminis $\mathrm{f}$. sp. tritici in wheat-growing areas of Mexico during 1988 and 1989. Plant Dis. 75:790-794.

Singh R P, S Rajaram (1991) Resistance to Puccinia recondita f. sp. tritici in 50 Mexican bread wheat cultivars. Crop Sci. 31:14721479.
Singh R P, R A McIntosh (1984) Complementary genes for reaction to Puccinia recondita tritici in Triticum aestivum I. Genetic and linkage studies. Can. J. Gen. Cytol. 26:723-735.

Singh R P, J Huerta-Espino, W Pfeiffer, P Figueroa-Lopez (2004) Occurrence and impact of a new leaf rust race on durum wheat in Northwestern Mexico from 2001 to 2003. Plant Dis. 88:703708.

Zhang H, D R Knott (1990) Inheritance of leaf rust resistance in durum wheat. Crop Sci. 30:1218-1222. 